\title{
Development of a driftless Johnson noise thermometer for nuclear applications
}

\author{
Jonathan V. Pearce ${ }^{1}$, Paul Bramley ${ }^{2}$, and David Cruickshank ${ }^{2}$ \\ ${ }^{1}$ National Physical Laboratory, Teddington, UK \\ ${ }^{2}$ Metrosol Ltd, Paulersbury, UK
}

\begin{abstract}
Existing temperature sensors such as thermocouples and platinum resistance thermometers suffer from calibration drift, especially in harsh environments, due to mechanical and chemical changes (and transmutation in the case of nuclear applications). A solution to the drift problem is to use temperature sensors based on fundamental thermometry (primary thermometers) where the measured property is related to absolute temperature by a fundamental physical law. A Johnson noise thermometer is such a sensor and uses the measurement of the extremely small thermal voltage noise signals generated by any resistive element to determine temperature using the JohnsonNyquist equation. A Johnson noise thermometer never needs calibration and is insensitive to the condition of the sensor material, which makes it ideally suited to long-term temperature measurement in harsh environments. These can include reactor coolant circuits, in-pile measurements, nuclear waste management and storage, and severe accident monitoring. There have been a number of previous attempts to develop a Johnson noise thermometer for the nuclear industry, but none have achieved commercialization because of technical difficulties. We describe the results of a collaboration between the National Physical Laboratory and Metrosol Limited, which has led to a new technique for measuring Johnson noise that overcomes the previous problems that have prevented commercialization. The results from a proof-of-principle prototype that demonstrates performance commensurate with the needs of nuclear applications is presented, together with details of progress towards the commercialization of the technology. The development partners have effected a step change in the application of primary thermometry to industrial applications and seek partners for field trials and further exploitation.
\end{abstract}

Keywords-Thermometry, Johnson noise, Johnson noise thermometry, traceability, ITS-90, temperature measurement

\section{INTRODUCTION}

HERMOMETRY in nuclear processes generally relies on

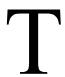

conventional sensors such as thermocouples or resistance thermometers. These thermometers need to be calibrated [1], and as the sensor material changes in harsh operating environments, the calibration also changes in an unknown manner [2,3]. In general calibration drift corresponding to between tens and hundreds of ${ }^{\circ} \mathrm{C}$ can be expected [4,5]. Such

Manuscript received 10 April 2019. This work was supported in part by Innovate UK and the UK government's Department for Business, Energy and Industrial Strategy.

Jonathan Pearce is with the National Physical Laboratory, Hampton Road, Teddington, TW11 0LW, United Kingdom (telephone: +44 (0)20 89436886 , e-mail: jonathan.pearce@npl.co.uk) drift leads to uncertainty about the process temperature, higher operating costs (frequent and costly sensor recalibrations/replacements), lower generating efficiencies (processes are operated at lower temperatures to ensure adequate safety margins) and compromises safety (temperature uncertainty being critical to the safe monitoring and control of reactors and other processes).

Johnson noise thermometry (JNT) has long been considered as a suitable solution to this problem [6]. JNT relies on the measurement of the electrical noise arising from the random thermal motion of charge carriers (electrons) in a sensor [7], and is completely independent of any material degradation. It is in a class of thermometry techniques called 'primary' thermometry [8], in that it can provide a direct measure of the temperature without recourse to calibration. As no calibration is required, it also does not experience calibration drift. JNTs exist mainly in National Measurement Institutes for extremely accurate metrology applications [6] such as measurement of the Boltzmann constant $[9,10]$ for the redefinition of the kelvin $[11,12]$, but these devices are large, slow, impractical, costly, and do not have sufficient immunity to electromagnetic interference (EMI). A JNT has also been applied to nuclear environments [13] which was successful, but slow, and it was not commercialized.

The reason JNT has not become widespread, despite the application of considerable ingenuity $[14,15]$, is because there are a number of significant technical challenges [6], the most demanding of which is the measurement of the very small signals in the harsh electromagnetic environments encountered in industry. The main goal of the current development work is to resolve the technical challenges that have so far prevented successful deployment of this technique in the nuclear power industry.

We describe a collaboration between Metrosol and NPL to design, build, test for electromagnetic compability (EMC) immunity to the conditions expected in the heavy industrial environment found in nuclear power stations, and metrologically validate a practical JNT. The aim is to provide a technology platform to facilitate the development, with industry partners, of products tailored for the specific technical and regulatory requirements of the industry.

Paul Bramley is with Metrosol Limited, Plum Park Estate, Watling Street, Paulerspury, Northamptonshire, NN12 6LQ, United Kingdom (telephone: +44 (0)1327 810284, e-mail: paul.bramley@metrosol.co.uk)

David Cruickshank is with Metrosol Limited, Plum Park Estate, Watling Street, Paulerspury, Northamptonshire, NN12 6LQ, United Kingdom (telephone: +44(0)1327 810284, e-mail: david.cruickshank@metrosol.co.uk) 
As newcomers to the industry, Metrosol and NPL seek partners with whom to collaborate and eventually license the core technology, to provide a faster and more effective route to market.

A first description of the practical Johnson noise thermometer was presented at the ANIMMA 2015 conference $[16,17]$ and some more recent developments have been presented in [18]. In this paper we summarise the key developments since 2015.

\section{PRACTICAL JNT}

The proposed JNT technology makes use of the Nyquist equation [19] to relate the mean noise voltage $V_{\mathrm{T}}$ measured across a resistor to the absolute temperature $T$ :

$$
\overline{V_{T}}=\sqrt{4 k T R \Delta f}
$$

where $k$ is Boltzmann's constant, $R$ is its resistance, and $\Delta f$ is the bandwidth. This allows the temperature to be determined regardless of the physical properties of the sensor (e.g. if sensor damage changes the resistance, there is no effect on the temperature measurement because resistance is continuously measured). The main difficulty is the magnitude of the noise voltage: even with a bandwidth of $1 \mathrm{MHz}$, a $1 \mathrm{k} \Omega$ resistor will generate an RMS voltage of just $1 \mu \mathrm{V}$, which is at the limit of current precision measurement capabilities.

The technical challenge is to measure this extremely small signal in an industrial environment where sensors may be exposed to interference signals up to $10 \mathrm{~V} / \mathrm{m}$ over the entire frequency band from direct current (DC) to several GHz. The current approach employs the established technique of using correlators to extract the required signal while rejecting unwanted noise, but new technologies have been developed for amplification and for providing a reference noise source. To do this, a more modest two sigma uncertainty (about $0.28^{\circ} \mathrm{C}$ ) is exchanged for much faster response times (about $6.5 \mathrm{~s}$ ).

A key difficulty with JNT is the fact that the Nyquist equation cannot be used directly because the bandwidth $\Delta f$ cannot be known. To overcome this, traditional JNTs switch between a reference noise source with Johnson noise voltage $V_{0}$, source resistance $R\left(T_{0}\right)$ at a reference temperature $T_{0}$ with bandwidth $\Delta f_{0}$, and another Johnson noise source $V_{\mathrm{T}}$, source resistance $R(T)$ at an unknown temperature $T$ with bandwidth $\Delta f[20]$. The switching eliminates spurious effects due to time dependent drifts in the gain and bandwidth of the amplifiers and filters [21]. The unknown temperature is then given by expressing the Nyquist equation in ratio form:

$$
T=\frac{\overline{V_{T}^{2}}}{V_{0}^{2}} \frac{R\left(T_{0}\right)}{R(T)} T_{0}
$$

There are several key factors which limit the accuracy of the switched-input correlation approach [20]. These are:

- The random nature of the noise signal, which necessitates averaging of the signal for a long enough period with a sufficiently wide bandwidth to reduce statistical fluctuations to the desired level. The time and bandwidth needed to achieve a given fractional uncertainty is given by Rice [22-24] and amounts to about $10 \mathrm{~s}$ to achieve an uncertainty of $0.1 \%$ (i.e. about $1{ }^{\circ} \mathrm{C}$ ) with a bandwidth of $100 \mathrm{kHz}$. A key development in the new JNT system is the use of higher resistances that therefore generate more Johnson noise and correspondingly reduces the measurement needed to achieve a given uncertainty.

- Electronic component nonlinearity: the tiny signal requires very high amplifier gain and wide bandwidths, and the thermometer must be operated as a comparator, comparing the noise powers from the reference arm at $T_{0}$ and the sensing arm at $T$. These nonlinearities vary with time and ambient temperature, and cannot be calculated or measured. To minimize errors, the same correlated and uncorrelated noise powers are needed in the two arms of the comparator, which means the sensing resistances must be chosen so that $R\left(T_{0}\right) T_{0}=R(T) T$.

- Transmission line effects: the preamplifiers must be located some distance from the sensors, which means that the transfer function of the transmission line is significant. The problem is the interaction of the sensor resistance with the transmission line, which results in a dependence of the transmission line frequency response on the sensor resistance. This causes an error that is most simply eliminated by employing a constant sensing resistance $R\left(T_{0}\right)=R(T)$. This requirement conflicts directly with the previous one needed to reject nonlinearity. Limiting the bandwidth can reduce the transmission line error, but in practice it still limits the achievable accuracy - furthermore, limiting the bandwidth increases the measuring time needed for a given accuracy.

Another way of looking at this is to study the assumption $\Delta f=\Delta f_{0}$ required by Equation (2), since as we switch between the two measurements there will inevitably be a change in the frequency response of the measurement system. The dominant effect is the capacitance of the cables ( $C$ for the sense resistor cable connected to $R, C_{0}$ for reference resistor cable connected to $R_{0}$ ) connecting the sensor and reference resistors to the input switch [25]. In order for the bandwidths to match, it is required that $R C=R_{0} C_{0}$. This can be met for a particular situation by trimming the circuit (e.g. by adding capacitance to one set of cables to balance the system). However, if either resistor changes value with time or temperature, there will be a mismatch in the effective bandwidths, leading to measurement errors. This means that the system would be susceptible to changes in a property of the sensor - the very problem we are trying to solve. Prior JNTs have solved this by ensuring both $\mathrm{RC}$ and $\mathrm{R}_{0} \mathrm{C}_{0}$ are much less than $1 / \Delta f$ so that the measurement is made on the flat part of the system frequency response 


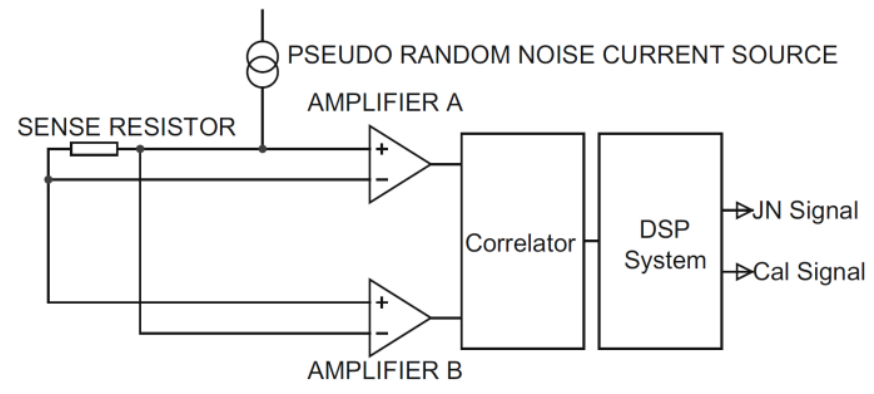

Fig. 1. New JNT topology.

affected by these impedances. This limited sensing resistors to around $100 \Omega$ and bandwidths to around $100 \mathrm{kHz}$, which in turn limits the size of the Johnson noise signal.

A way around these issues is to replace the reference resistor with a cryogenic quantized voltage noise source based on Josephson junction arrays [26] or, more practically, a white pseudo-random noise (PRN) voltage source [24]. The digitally synthesized PRN signal is the sum of a number of sinewaves (of equal amplitude) that are equally spaced in frequency and with random phase. This generates a Gaussian noise-like waveform that will repeat with a period equal to the reciprocal of the frequency spacing. The waveform can be stored as digital samples and then recreated by streaming the sampled waveform to a digital-to-analogue converter (DAC). The 'comb' of tones comprising the PRN calibration signal then samples the frequency response of the measurement system including tracking changes over time.

\section{NEW JNT MEASUREMENT TOPOLOGY}

We have seen that a large number of problems stem from switching between a known white noise reference signal and the unknown Johnson noise signal. A new measurement topology was therefore devised to overcome the need to switch, as shown in Fig. 1.

The calibration signal (a PRN waveform) is injected as current into the measurement circuit to generate a calibration voltage signal in the sensor resistor; this is of course overlaid with the Johnson noise signal. The calibration signal and the Johnson noise signal both experience the same frequency response as they pass through the connecting cables, amplifiers and correlator. The Johnson noise and calibration signals are then separated from the composite signal by a digital signal processing (DSP) system. The ratio of the Johnson noise and calibration signal can then be calculated in order to determine the absolute Johnson noise power and hence the temperature of the sense resistor.

Importantly, this topology is tolerant of a 'non-flat' frequency response since the two signals experience the same frequency response. As a result, it is possible to use a much wider bandwidth ( $1 \mathrm{MHz}$ or more), including frequencies where there is significant attenuation arising from the connecting cables. In the current realization, the PRN signal comprises tones with randomized phase at $152 \mathrm{~Hz}$ intervals between $10 \mathrm{kHz}$ and 1.2 MHz. Lower frequency tones are used to determine the resistance of the sense resistor using Ohm's law, since the attenuation by the cable capacitance is negligible at these frequencies. In addition to the current tones, one other piece of information is needed to calculate resistance, namely amplifier gain at low (i.e. flat) frequency, or voltage tones.

For practical reasons, the calibration current is injected at a point adjacent to one of the pre-amplifier outputs. Fig. 2 shows this arrangement after folding out the circuit in Fig. 1.

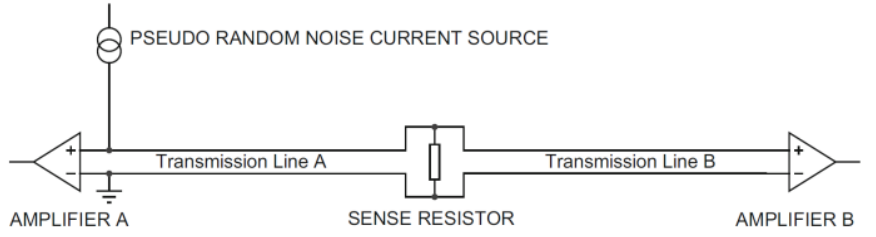

Fig. 2. Transmission lines and current injection for the new Johnson noise measurement topology.

A lumped circuit model of the effect of the transmission lines on the signal attenuation and resulting measurement error was developed, to enable the exploration of parameter space for the system. The errors reduce as the value of the sense resistor is increased. Fig. 3 shows the effect of increasing the sensor resistance for a cable with total series resistance of $1 \Omega$, transmission lines of capacitance $0 \mathrm{pF}$ to $100 \mathrm{pF}$ and bandwidths of $0 \mathrm{MHz}$ to $1 \mathrm{MHz}$.
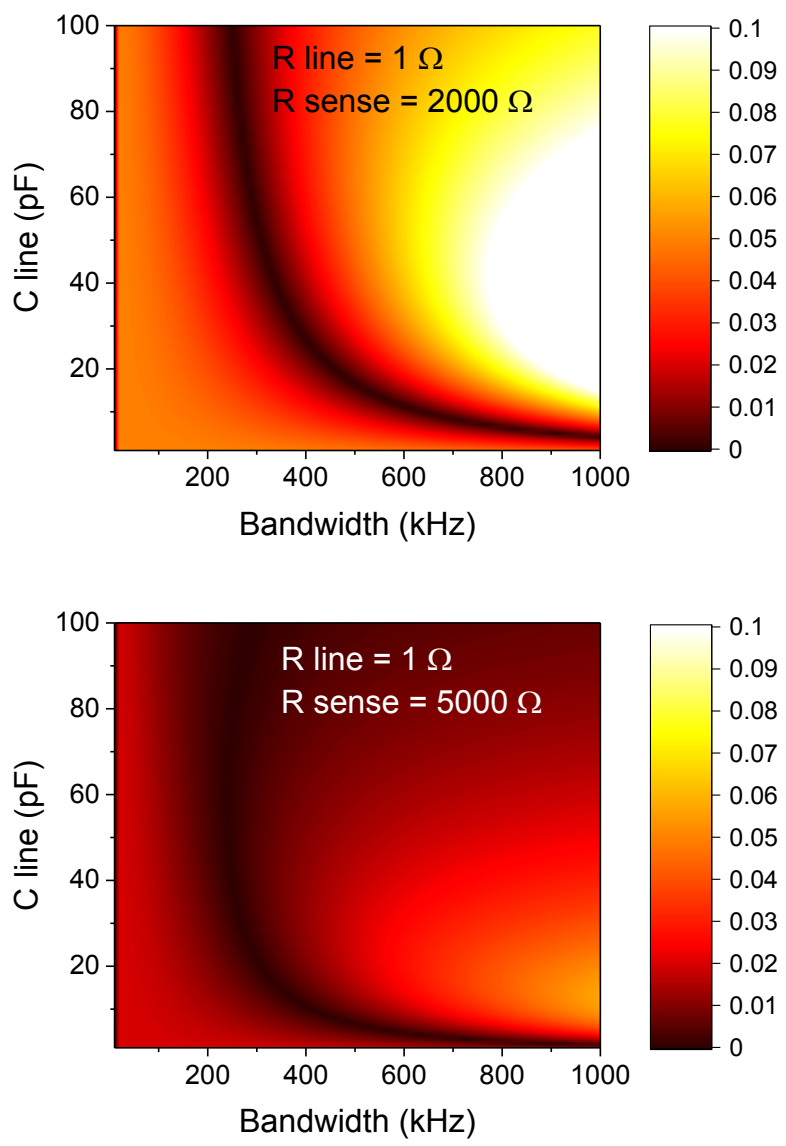

Fig. 3. Effect of sensor resistance on the measurement error, for two different values of the sense resistance [18]. Colour corresponds to the magnitude of the error, ranging from $0 \%$ (blue) to $0.1 \%$ (red).

Fig. 3 shows the benefit of using a higher sense 
resistance. Increasing the sensor resistance also increases the magnitude of the Johnson noise signal.

As we can measure the frequency response using the tones, we are also able to use a much higher bandwidth as we are no longer limited to operating on the flat part of the input circuit's frequency response. Overall, the ability to use a high value resistor and the consequent increased Johnson noise signal, the ability to access a higher bandwidth, and the lack of switching mean that the measurement time for a given uncertainty is minimized. In the current implementation this reduces the measurement time by a factor of $\sqrt{ } 20$ over prior JNTs (1 MHz c.f. $100 \mathrm{kHz}$ bandwidth and a factor of two for not switching).

The inventions described above are subject to a filed patent, GB2545176 [27].

\section{RESULTS}

The first benchtop prototype (JNT1) and the second, more compact prototype (JNT2) are shown in Fig. 4. The device consists of a long probe with a similar external aspect to thermocouples or resistance thermometers, and a 'sensing head' containing the sensing electronics. The probe length is important as longer probes will increase cable capacitance and cause low pass filtering, and this will limit the available bandwidth. For example, a $0.5 \mathrm{~m}$ probe may achieve a bandwidth of around $1 \mathrm{MHz}$, while a $5 \mathrm{~m}$ probe of the same construction would only achieve a bandwidth of around $100 \mathrm{kHz}$. This would increase the measurement time needed to achieve the same uncertainty by a factor of $\sqrt{ } 10$.

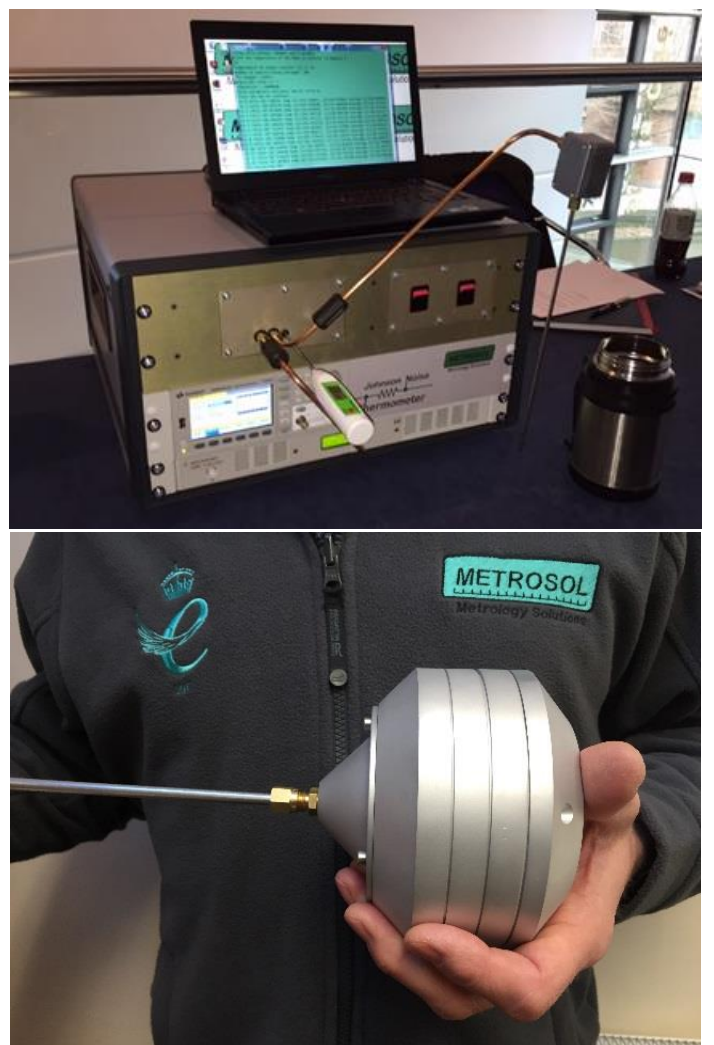

Fig. 4. Prototype Johnson noise thermometers JNT1 (top) and JNT2 (bottom).
Initial tests were conducted on JNT1 to ensure that the measured spectra were clean with no evidence of contamination from external noise sources. Early results with JNT1 showed contaminated spectra and highlighted the sensitivity of the system to external interference (Fig. 5, top panel). This problem was left unresolved in previous work [14]. Considerable effort has been devoted to improving cabling, grounding and shielding. In particular, the sensitive parts of the circuit were modified to use a full tri-axial probe connection. Following these modifications, the spectra became consistently 'clean', as exemplified by the spectrum from JNT2 in Fig. 5, bottom panel.
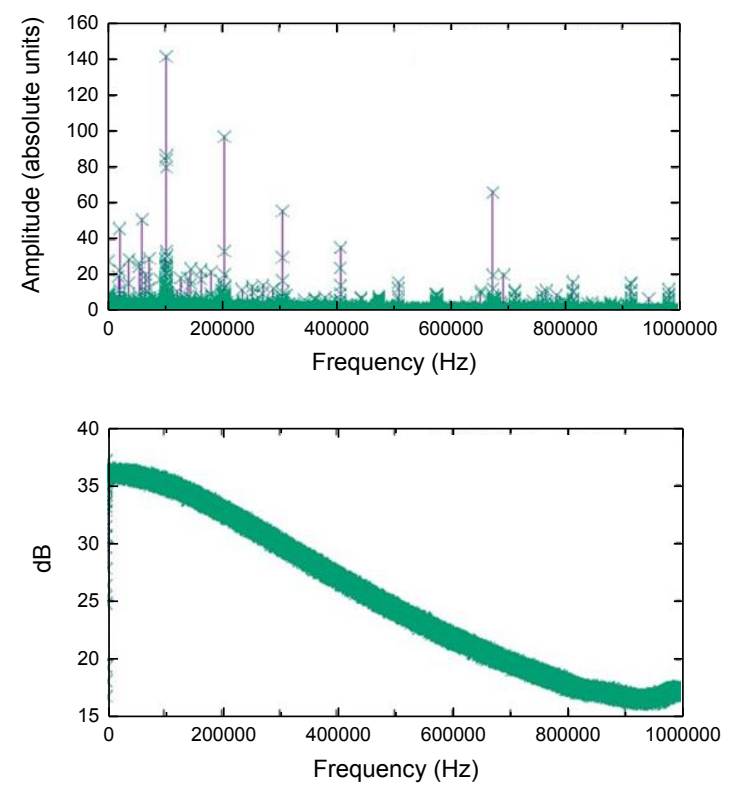

Fig. 5. A very early spectrum from JNT1 showing heavy contamination from EMI (top) and a later spectrum after dramatic circuit improvements in JNT2 (bottom).

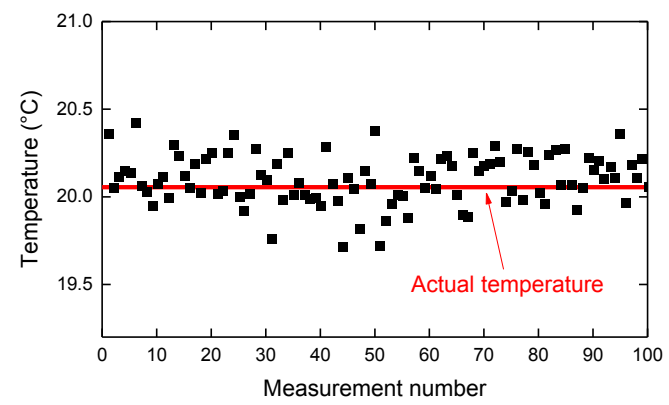

Fig. 6. 100 measurements of temperature made with the JNT over three days (JNT 1 prototype).

The JNT1 system was extended to include the calibration tones allowing the absolute power and hence the temperature of the sensor resistor to be determined. The temperature of a $5 \mathrm{k} \Omega$ foil resistor was then measured in an oil bath maintained at $20.06{ }^{\circ} \mathrm{C} \pm 0.01{ }^{\circ} \mathrm{C}$, as measured with a traceably calibrated reference thermometer. A series of 100 readings were taken over a three day period as shown in Fig. 6. The standard deviation of the temperature determined using the Johnson noise measurements was found to be $0.14{ }^{\circ} \mathrm{C}$. 
The much improved JNT2 device was submitted for EMC testing applicable to heavy industrial environments. The radiated field immunity was tested to EN 61000-4-3 [28]. Field strengths were $10 \mathrm{~V} \mathrm{~m}^{-1}$ from $80 \mathrm{MHz}$ to $1000 \mathrm{MHz}$ and $3 \mathrm{~V} \mathrm{~m}^{-1}$ from $1 \mathrm{GHz}$ to $2.7 \mathrm{GHz}$; in both frequency ranges there was no observable effect on the thermometer. It was also tested for radiated emissions to EN 55011 [29] (from $30 \mathrm{MHz}$ to 1000 $\mathrm{MHz}$ ) and showed no detectable emissions. This represents an extremely important milestone in the development of a practical JNT; insufficient immunity to EMC thwarted previous efforts.

The JNT2 is currently a compact, self-contained thermometer capable of making temperature measurements without any a-priori calibration, and has extremely high immunity to EMC. As such it represents a step change improvement over the prototype plans presented at the ANIMMA 2015 conference. In terms of the Technology Readiness Level (TRL) [30], in the last 4 years it has jumped from TRL 2 (Technology concept and/or application formulated) to TRL 5 (Component and/or breadboard critical function verification in relevant environment).

\section{CONCLUSION}

The continuing development of a practical Johnson noise thermometer (JNT), as part of a joint activity between NPL and Metrosol Limited, has been described. The development, characteristics and performance have been described. The JNT measures the voltage arising from the thermal motion of charge carriers, which are related to temperature through fundamental physics. Importantly, the temperature measurement is unaffected by changes in the property of the sensor (except the resistance which can be measured simultaneously with the temperature) and is independent of any calibration. This opens up the prospect of truly 'driftless' temperature sensing which has substantial benefits for the measurement and control of processes needing reliable temperature measurement. Over the last 3 years the JNT has undergone a step change in size and EMC resilience, and currently measures temperature with an uncertainty (two sigma) of about $0.28^{\circ} \mathrm{C}$ with a measuring time of about $6.5 \mathrm{~s}$.

\section{REFERENCES}

[1] J.V. Pearce, "Extra points for thermometry", Nat. Phys. Vol. 13, January 2017, p. 104

[2] M.J. Kelly, W.W. Johnston and C.D. Baumann, "The Effects of Nuclear Radiation on Thermocouples", in Temperature: Its Measurement and Control in Science and Industry (TMCSI), Vol. 3 part 2, pp. 265-269, ed. Herzfeld, Reinhold Publishing Corp, NY, 1962

[3] W.E. Browning and C.E. Miller, Calculated Radiation Induced Changes in thermocouple Composition, Temperature: Its Measurement and Control in Science and Industry (TMCSI), Vol. 3 part 2, pp. 271-276, ed. Herzfeld, Reinhold Publishing Corp, NY, 1962

[4] H.M. Hashemian and Jun Jiang, "Nuclear Plant Temperature Instrumentation", Nuclear Engineering and Design 239, 3132-3141 (2009)

[5] J.L. Rempe, D.L. Knudson, K.G. Condie, S. Curtis Wilkins, "Evaluation of Specialized Thermocouples for High-Temperature In-Pile Testing. Proceedings of ICAPP '06 Reno, nV USA, June 4-8, 2006, paper 6086 [6] D.R. White, R. Galleano, A. Actis, H. Brixy, M. De Groot, J. Dubbeldam, A.L. Reesink, F. Edler, H. Sakurai, R.L. Shepard, J.C. Gallop, "The status of Johnson noise thermometry", Metrologia 33, 325-
$335(1996)$

[7] J.B. Johnson, Thermal agitation of electricity in conductors. Nature 119, 50-51 (1927)

[8] M.R. Moldover, W.L. Tew, H.W. Yoon, "Advances in thermometry", Nat. Phys., 12, 7-11 (2016)

[9] S.P. Benz, A. Pollarolo, J. Qu, H. Rogalla, C. Urano, W.L. Tew, P.D. Dresselhaus, D.R. White, "An electronic measurement of the Boltzmann constant", Metrologia, 48, 142 (2011)

[10] J. Qu, S.P. Benz, K. Coakley, H. Rogalla, W.L. Tew, R. White, K. Zhou, Z. Zhou, "An improved electronic determination of the Boltzmann constant by Johnson noise thermometry", Metrologia, 54, 549 (2017)

[11] M. de Podesta, Rethinking the kelvin, Nat. Phys. 12, 104 (2016)

[12] G. Machin, The kelvin redefined, Meas. Sci. Technol. 29022001 (2018)

[13] H. Brixy, R. Hecker, J. Oehmen, "Temperature measurements in the high-temperature range $\left(1000-2000^{\circ} \mathrm{C}\right)$ by means of noise thermometry", High Temperature High Pressure, 23, 625-631, 1991

[14] Roger A. Kisner et al. 2005. Development of a Johnson Noise Thermometer for Nuclear Power Use, Final Report of the International Nuclear Energy Research Initiative subtask 2.2: Development of

Enhanced Reactor Operation Strategy Through Improved Sensing and Control at Nuclear Power Plants

[15] H. Brixy, R. Hecker, J. Oehmen, Temperature measurements in the hightemperature range $\left(1000-2000{ }^{\circ} \mathrm{C}\right)$ by means of noise thermometry, High Temperature High Pressure, 23, 625-631, 1991

[16] M. Giot, L. Vermeeren, A. Lyoussi, C. Reynard-Carette, C. Lhuillier, P. Mégret, F. Deconinck, B.S. Gonçalves, "Nuclear instrumentation and measurement: a review based on the ANIMMA conferences", EPJ Nuclear Sci. Technol. 3, 33 (2017)

[17] J.V. Pearce, A. Greenen, P. Bramley, D. Cruickshank, "Towards a practical Johnson noise thermometer for long-term measurements in harsh environments", proc. 2015 4th International Conference on Advancements in Nuclear Instrumentation Measurement Methods and their Applications (ANIMMA) DOI: 10.1109/ANIMMA.2015.7465506 (2016)

[18] P. Bramley, D. Cruickshank, J.V. Pearce, "The development of a practical, drift-free, Johnson noise thermometer for industrial applications", Int. J. Thermophys. 38, 25 (2017)

[19] H. Nyquist, "Thermal Agitation of Electric Charge in Conductors", Phys. Rev. 32, 110-113 (1928)

[20] S.P. Benz, J.M. Martinis, S.W. Nam, W.L. Tew, D.R. White, “A New Approach to Johnson Noise Thermometry using a Josephson Quantized Voltage Source for Calibration”, Proceedings of TEMPMEKO 2001: 8th International Symposium on Temperature and Thermal Measurements in Industry and Science, 19-21 June 2001, Berlin, Germany

[21] D.R. White, S.P. Benz, "Constraints on a synthetic-noise source for Johnson noise thermometry", Metrologia 45, 93-101 (2008)

[22] S. O. Rice, "Mathematical Analysis of Random Noise", Bell Syst. Tech. J., 23, 282-332 (1944)

[23] S.O. Rice, "Mathematical analysis of random noise", Bell Syst. Tech. J. 24, 46-156 (1945)

[24] D.R. White et al., "Measurement time and statistics for a noise thermometer with a synthetic-noise reference", Metrologia 45, 395 (2008) [25] D.R. White and J.-F. Qu, "Frequency-response mismatch effects in Johnson noise thermometry", Metrologia 55(1), 38 (2018)

[26] S.W. Nam et al., "Johnson noise thermometry measurements using a quantized voltage noise source for calibration", IEEE Trans. Instrum. Meas. 52, 550-554 (2003)

[27] https://www.ipo.gov.uk/p-ipsum/Case/PublicationNumber/GB2545176 [28] EN 61000-4-3: Electromagnetic compatibility (EMC) - Part 4-3: Testing and measurement techniques - Radiated, radio-frequency, electromagnetic field immunity test

[29] EN 55011: Industrial, scientific and medical equipment — Radiofrequency disturbance characteristics - Limits and methods of measurement [30] ISO 16290:2013, Space systems - Definition of the Technology Readiness Levels (TRLs) and their criteria of assessment 